\title{
The dense ring in the Coalsack: the merging of two subsonic flows?
}

\author{
J. M. Rathborne, C. J. Lada and W. Walsh \\ Harvard-Smithsonian Center for Astrophysics, 60 Garden Street, Cambridge, MA 02138, \\ USA:jrathborne@cfa.harvard.edu,clada@cfa.harvard.edu,wwalsh@cfa.harvard.edu \\ and \\ M. Saul \\ School of Physics, The University of New South Wales, Sydney, NSW 2052, Australia: \\ msaul@phys.unsw.edu.au \\ and \\ H. M. Butner \\ Department of Physics and Astronomy, James Madison University, Harrisonburg, VA \\ 22807, USA: butnerhm@jmu.edu
}

\begin{abstract}
A recent high angular resolution extinction map toward the most opaque molecular globule, Globule 2, in the Coalsack Nebula revealed that it contains a strong central ring of dust column density. This ring represents a region of high density and pressure that is likely a transient and possibly turbulent structure. Dynamical models suggest that the ring has formed as a result of a sudden increase in external pressure which is driving a compression wave into the Globule. Here we combine the extinction measurements with a detailed study of the $\mathrm{C}^{18} \mathrm{O}(1-0)$ molecular line profiles toward Globule 2 in order to investigate the overall kinematics and, in doing so, test this dynamical model. We find that the ring corresponds to an enhancement in the $\mathrm{C}^{18} \mathrm{O}$ non-thermal velocity dispersion and non-thermal pressure. We observe a velocity gradient across the Globule that appears to trace two distinct systematic subsonic velocity flows that happen to converge within the ring. We suggest, therefore, that the ring has formed as two subsonic flows of turbulent gas merge within the Globule. The fact that the outer layers of the Globule appear stable against collapse yet there is no centrally condensed core, suggests that the Globule may be evolving from the outside in and has yet to stabilize, confirming its youth.
\end{abstract}


Subject headings: stars: formation - ISM: globules - ISM: kinematics and dynamics - ISM: molecules

\section{Introduction}

Understanding the initial conditions that lead to the formation of dense cores is important as it has direct implications for theories of star formation. Recent studies of starless cores reveal that the kinematics in the early stages of core collapse can be quite complex: in some cases the molecular tracers indicate simultaneously opposing motions (expansion and infall; Lada et al. 2003). In the case of the starless Bok globule B68, the presence of both inward and outward motions, traced by molecular line emission, has been interpreted to arise due to the oscillation of the outer layers of the cloud (Lada et al. 2003; Redman et al. 2006; Maret et al. 2007). This idea is strengthened by modeling that suggests that the complex motions within starless cores can occur due to oscillating perturbations about a thermally-supported, pressure bounded structure (Keto et al. 2006; Broderick et al. 2007). Indeed, recent numerical simulations reveal that small amounts of rotation can cause initially unstable and collapsing cores to stabilize and oscillate (Matsumoto \& Hanawa 2003).

The combination of high angular resolution extinction and molecular line observations toward cold, dense starless cores can trace the properties and kinematics of the dense gas and allow us to gain insight into the physical nature of core and, thus, star formation. Extinction measurements are particularly useful for tracing the internal structure of cores because they probe the material along the line-of-sight and are not susceptible to optical depth effects (as in the case of ${ }^{12} \mathrm{CO}$ molecular line emission) or assumptions about the dust temperature or emissivity (as in the case of millimeter continuum emission). Thus, the combination of dust extinction observations with optically thin molecular line observations can accurately reveal the masses and densities of the dust as well as the kinematics and dynamics of the gas.

In this paper we combine extinction observations with a detailed kinematic study of the molecular line profiles observed toward Tapia's Globule 2 in the Coalsack Nebula. Because Globule 2 does not contain a central protostar, is one of the densest cores within the Coalsack complex, and is thought to be in a very early stage of condensing, a detailed study of its kinematic structure could provide important details on the processes leading to core formation.

The Coalsack Nebula is a nearby dark molecular cloud complex that extends almost $6^{\circ}$

on the sky ( $\sim 15$ pc at its distance of 150 pc; Cambrésy 1999) and appears completely devoid of star formation activity (Nyman et al. 1989; Bourke et al. 1995; Kato et al. 1999). It has a 
total mass of $\sim 3550 M_{\odot}$ and is characterized by complex, filamentary molecular structures which contain many dark cores with kinetic temperatures, $\mathrm{T}_{K}$, of 8-11 K (Nvman et al. 1989). The darkest and densest of these cores are Tapia's Globules 1, 2, and 3 (Tapia 1973; Bok 1977).

Using deep near-infrared imaging observations obtained with the European Southern Observatory (ESO) 3.6m New Technology Telescope (NTT), Lada et al. (2004) were able to examine the structure of Globule 2 in significantly greater detail than previously possible. The NICE method (Lada et al. 1994; Alves et al. 1998) was used to generate a visual extinction $\left(\mathrm{A}_{V}\right)$ map that was constructed by measuring the individual infrared extinctions to approximately 24,000 stars located behind Globule 2. This resulted in an extinction map across the core with significantly improved angular resolution $\left(\sim 15^{\prime \prime}\right)$ compared to previous maps ( 60"; Jones et al. 1980, 1984; Racca et al. 2002).

The extinction map revealed Globule 2 to have a mass of $\sim 6.1 M_{\odot}$, a mean density of $\sim 3 \times 10^{3} \mathrm{~cm}^{-3}$ and Jeans mass, $M_{J}$, of $\sim 10.4 M_{\odot}$. From near-infrared polarimetry observations, Jones et al. (1984) suggested that Globule 2 is supported either by turbulence or a magnetic field and appears stable. This is also consistent with the fact that the derived mass is less than $M_{J}$ suggesting it is unlikely to fragment further. Moreover, a Bonnor-Ebert (BE) fit to the outer regions of the Globule's radial extinction profile suggests that it is globally stable against collapse (Lada et al. 2004). The Globule's derived BE mass is $\sim 6 M_{\odot}$. Even though this is comparable to the derived mass calculated from the dust, the high angular resolution infrared extinction map toward the Globule does not reveal a centrally condensed core as would be expected if it was a true BE sphere. Instead, the most prominent feature within the extinction map is a strong central ring of dust column density. This ring likely represents a transient structure that has formed from a region of high density and pressure (Lada et al. 2004). Because there is no source of internal pressure to support the ring against gravitational collapse, it is thought that the ring is not in dynamical equilibrium with its surroundings and, given the short timescales for inward collapse $\left(2 \times 10^{5}\right.$ years $)$, most likely represents an extremely early stage of evolution.

From a single $\mathrm{C}^{18} \mathrm{O}(2-1)$ spectrum toward the center of the globule, Lada et al. (2004) noted a double-peaked profile comprising a bright narrow line and a fainter broad line. Based on the absence of this double-lined profile in lower angular resolution spectra (e.g., Brooks et al. 1976; Nyman et al. 1989; Kato et al. 1999), it was suggested that the narrow line component most likely arises within a small region and that the broad line component traces a more spatially extended feature (Lada et al. 2004). The line width of each blended component was found to be super-thermal indicating that significant non-thermal, or turbulent, motions most likely dominate. Dynamical models for Globule 2, however, suggest that 
the ring structure has formed as a result of a sudden increase in external pressure which is driving a compression wave into the Globule (Hennebelle et al. 2006).

Using the Mopra 22-m telescope, we have obtained the first complete high angular and spectral resolution molecular line map of Globule 2. Because ${ }^{12} \mathrm{CO}$ is optically thick toward most dense cores, and is thus only tracing the outer layers, we choose to conduct this study using the optically thin $\mathrm{C}^{18} \mathrm{O}(1-0)$ transition which is far superior in tracing the dense gas associated with the central core. In combination with the extinction map, these data should allow us to test the various formation mechanisms for the ring feature; is it a high-density transient structure formed via the dissipation of turbulent gas (Lada et al. 2004) or does it trace the propagation of a compression wave through the Globule (Hennebelle et al. 2006)? Moreover, these data will enable the relative spatial distributions of the two molecular line components to be definitively traced. In particular, does the narrow-line component originate exclusively in the dense ring? Does the broad-line component primarily trace the turbulent envelope of the Globule? Answers to these questions will directly test the idea that Globule 2 is in perhaps the earliest stage of evolution: a dense core in the process of formation.

\section{Observations}

Molecular line maps of Globule 2 in the Coalsack Nebula were obtained with the Mopra 22-m telescope in 2006 July. The $8 \mathrm{GHz}$ spectrometer MOPS was used to simultaneously observe a number of transitions including $\mathrm{C}^{18} \mathrm{O}(1-0) 1$. The spectrometer was used in 'zoom' mode such that one spectral window covered each of the lines of interest. Each window was $137.5 \mathrm{MHz}$ wide and contained 4096 channels in both orthogonal polarizations. This produced a velocity resolution of $0.09 \mathrm{~km} \mathrm{~s}^{-1}$ for the $\mathrm{C}^{18} \mathrm{O}$ transition. At these frequencies the Mopra beam is $\sim 33^{\prime \prime}$ (Ladd et al. 2005).

The observations were obtained using the On-The-Fly (OTF) mode by scanning in both Right Ascension and Declination. All maps were Nyquist sampled. A fixed off-source position was used for all maps ( $\alpha=12: 29: 57.1, \delta=-63: 32: 21.8, \mathrm{~J} 2000)$. The nearby $\mathrm{SiO}$ maser, $\mathrm{R}$ Car, was used to check the pointing accuracy approximately every hour and was typically better than $9^{\prime \prime}$. The system temperatures were checked frequently using a paddle and were typically $180 \mathrm{~K}$.

A total of fourteen $5^{\prime} \times 5^{\prime}$ OTF maps were combined (using the system temperature

\footnotetext{
${ }^{1}$ The other transitions observed included ${ }^{12} \mathrm{CO},{ }^{13} \mathrm{CO}, \mathrm{HCO}^{+}, \mathrm{CH}_{3} \mathrm{OH}$, and $\mathrm{CS}$ and will be presented in future papers (Saul et al., Butner et al. in prep.).
} 
weighted median) to produce the final map. The data were initially baseline subtracted and converted to a temperature scale using the $\mathrm{AIPS}^{++}$task livedata. All raw data are in the

$\mathrm{T}_{A}^{*}$ scale. To convert to the main beam brightness, $\mathrm{T}_{m b}$, we use the beam efficiencies listed in Ladd et al. (2005).

The OTF maps were combined and gridded into data cubes using the AIPS $^{++}$task gridzilla. To produce the final map, a Gaussian smoothing kernel with a FWHM of $0.55^{\prime}$ was applied to the data. This resulted in the final cube having an effective angular resolution of $\sim 46^{\prime \prime}$. The final $\mathrm{C}^{18} \mathrm{O}$ map has a $\mathrm{T}_{A}^{*}$ rms noise sensitivity of $0.08 \mathrm{~K}$ channel $^{-1}$.

\section{Results}

Figure 1 shows the visual extinction map of Globule 2 (15" angular resolution). This map was generated from deep near-infrared images by measuring the extinction toward $\sim 24,000$ background stars (Lada et al. 2004). The ring is clearly seen as an extinction enhancement surrounding a central depression. Overlaid on this map is the $\mathrm{C}^{18} \mathrm{O}(1-0)$ integrated emission obtained with the Mopra 22-m telescope (the emission was integrated over the velocity range of -7.0 to $-5.0 \mathrm{~km} \mathrm{~s}^{-1} ; 46^{\prime \prime}$ angular resolution). These data show that the $\mathrm{C}^{18} \mathrm{O}$ integrated emission follows the overall morphology of the extinction. There are noticeable differences, however. The $\mathrm{C}^{18} \mathrm{O}$ integrated emission does not trace completely the extinction ring. In particular, it appears that the extinction peaks toward the eastern arc of the ring, whereas the $\mathrm{C}^{18} \mathrm{O}$ integrated emission peaks toward western arc. Some of these differences may arise due to the different angular resolutions of the two datasets $\left(15^{\prime \prime}\right.$ and $45^{\prime \prime}$ respectively). When smoothed to an angular resolution of $45^{\prime \prime}$, the extinction image shows a much shallower contrast between the ring and the central hole.

Figure 2 shows the $\mathrm{C}^{18} \mathrm{O}(1-0)$ channel maps across the Globule overlaid with contours of the smoothed visual extinction image (the $A_{V}$ map of Fig. 1 was smoothed to $46^{\prime \prime}$ to match the angular resolution of the $\mathrm{C}^{18} \mathrm{O}$ data). A clear velocity gradient is evident across the Globule.

\subsection{Gaussian component analysis}

The individual $\mathrm{C}^{18} \mathrm{O}(1-0)$ spectra across the Globule reveal that the emission profile differs quite considerably on and off the extinction ring. Based on the detection of a 'narrow-line' ( $\left.\mathrm{v}_{L S R} \sim-5.64 \mathrm{~km} \mathrm{~s}^{-1}\right)$ and 'broad-line' $\left(\mathrm{v}_{L S R} \sim-5.83 \mathrm{~km} \mathrm{~s}^{-1}\right.$ ) component within the $\mathrm{C}^{18} \mathrm{O}(2-1)$ spectrum toward the ring and the absence of the narrow-line component 
within lower-angular resolution surveys, it was suggested that the broad line was spatially extended across the Globule while the narrow line was restricted to the extinction ring (Lada et al. 2004). Thus, to characterize and separate these two emission features and determine any spatial differences between them, we fit Gaussian profiles to each spectrum within the map.

Because we expect the broad-line component to contribute to the observed spectra at all points across the map, but the narrow-line component to only those spectra coincident with the extinction ring, we attempt to fit both a one- and two-component Gaussian profile to each spectrum within the map. Initial guesses for the $\mathrm{v}_{L S R}$, line width, and peak temperature for the Gaussian profiles were based on the observed profiles from Lada et al. (2004). All were free parameters2. For example, when fitting a one-component Gaussian profile we use as input the values determined for the 'broad' line. When fitting a two-component Gaussian profile we input the values derived for both the 'broad' and 'narrow' lines. At each position within the map we select either the one- or two-component Gaussian fit based on which had the lower residuals. We then assigned each fitted Gaussian profile to either the 'narrow' or 'broad' line component based on its derived $\mathrm{v}_{L S R}$. We find that $\sim 70 \%$ of the spectra were well fit by a one-component Gaussian profile, while the remaining $\sim 30 \%$ were best fit by two components.

As expected, we find that the spectra toward the extinction ring are best matched by a two-component Gaussian profile, while the spectra external to the ring are best matched by a single component. Contrary to the original prediction, however, the central $\mathrm{v}_{L S R}$ derived for the emission external to the ring does not correspond to a single $\mathrm{v}_{L S R}$, nor does it simply correspond to the 'broad' component. Instead, the $\mathrm{v}_{L S R}$ shows a range of values (from -6.0 to $-5.7 \mathrm{~km} \mathrm{~s}^{-1}$ ) which suggests that both the 'broad' and 'narrow' lines are located external to the ring. It appears, therefore, that the narrow-line component may not simply arise within the ring, but instead may correspond to a spatially separate velocity component extended across the Globule.

This can be seen in the $\mathrm{C}^{18} \mathrm{O}$ channel maps of Figure 2; the component at $\sim-5.64 \mathrm{~km} \mathrm{~s}^{-1}$ peaks in the ring, but also extends beyond it. The broad-line component $\left(\sim-5.82 \mathrm{~km} \mathrm{~s}^{-1}\right)$ also arises both in and out of the ring, the only difference being it appears to trace the molecular material on the opposing side of the extinction ring to the narrow-line component. Figure 3 shows the measured peak temperature $\left(\mathrm{T}_{m b}\right)$, central velocity $\left(\mathrm{v}_{L S R}\right)$, and one-dimensional velocity dispersion $(\sigma)$ for the two components from the Gaussian fitting procedure. We find that the individual measured central $\mathrm{v}_{L S R}$ and $\sigma$ for the two components

\footnotetext{
${ }^{2}$ the Gaussian fitting was performed within IDL using the gaussfits procedure.
} 
show a dispersion in their values. This suggests that the kinematics of the region are quite complex and not well matched simply by two Gaussian $\mathrm{v}_{L S R}$ components toward the ring and a single $\mathrm{v}_{L S R}$ component external to it.

The absence of the narrow-line component in previous molecular line surveys is not surprising given their limited angular and velocity resolutions. Taking an average $\mathrm{C}^{18} \mathrm{O}(1-$ 0 ) spectra over the region contained within the beam of the $\mathrm{H}_{2} \mathrm{CO}$ observations (4.4; Brooks et al. 1976), we find that the molecular line profile is characterized by a single emission line, with $\mathrm{v}_{L S R} \sim-5.8 \mathrm{~km} \mathrm{~s}^{-1}$ and line width, $\Delta \mathrm{V}$, of $0.6 \mathrm{~km} \mathrm{~s}^{-1}$.

\subsection{Moment analysis}

Rather than refer to the individual components as the 'narrow' and 'broad' lines based on their original $\mathrm{v}_{L S R}$ and Gaussian identification, these data reveal that the $\mathrm{C}^{18} \mathrm{O}$ emission displays complex velocity features across the Globule. Thus, in this section we characterize the emission via a moment analysis which is independent of Gaussian deconvolution and assumptions about individual components.

Over the extent of the Globule, the combination of these velocity features manifest themselves as a velocity gradient. This velocity gradient is seen in the $\mathrm{C}^{18} \mathrm{O}$ channel maps (Fig. 21). While the brightest emission coincides with the extinction ring, we also see the redand blue-shifted emission tending to arise on opposite sides of the extinction ring. This can also be seen in the position-velocity, $(\ell-V)$, diagrams of Figure 4 . The left panel shows the $(\ell-V)$ diagram averaged over the extinction ring in Declination (the emission was averaged over $\Delta \delta$ of $6^{\prime}$ centered on the ring). The right panel shows the Right Ascension-averaged diagram (the emission was averaged over $\Delta \alpha$ of $18^{\prime}$ centered on the ring). The velocity gradient is more apparent in the Declination-averaged $(\ell-V)$ diagram.

The zeroth moment map, or integrated intensity image, is defined as

$$
I=\int T_{m b}(v) d v
$$

where $\mathrm{T}_{m b}(v)$ is the main beam brightness at a given velocity $v$. The image was generated by integrating all of the emission over the range of -7.0 to $-5.0 \mathrm{~km} \mathrm{~s}^{-1}$ and is shown in Figure 5 overlaid with contours of the smoothed visual extinction image. The extinction ring is clearly seen as an enhancement in $\mathrm{C}^{18} \mathrm{O}$ emission.

The first moment map, $\mathrm{M}_{1}$, is a measure of the intensity (in this case $\mathrm{T}_{m b}$ ) weighted 
velocity field and is defined as

$$
M_{1}=\frac{\int T_{m b}(v) v d v}{\int T_{m b}(v) d v}
$$

(Sault et al. 1995). The $\mathrm{C}^{18} \mathrm{O}$ first moment map is also shown in Figure 5 and clearly shows the aforementioned velocity gradient. Toward the edges of the extinction ring the velocity

field ranges from -6.0 to $-5.7 \mathrm{~km} \mathrm{~s}^{-1}$ Toward the peak of the extinction ring, the $\mathrm{v}_{L S R}$ of the emission is $\sim-5.8 \mathrm{~km} \mathrm{~s}^{-1}$.

The intensity weighted velocity dispersion is represented via the second moment, $\mathrm{M}_{2}$, which is defined as

$$
M_{2}=\sqrt{\frac{\int T_{m b}(v)\left(v-M_{1}\right)^{2} d v}{\int T_{m b}(v) d v}}
$$

The $\mathrm{C}^{18} \mathrm{O}$ second moment map is also shown in Figure 5. We see a clear enhancement in the velocity dispersion toward the extinction ring. Moreover, the morphology of the velocity dispersion and the $\mathrm{A}_{V}$ appear to match very well. Indeed, of all the measured quantities, the velocity dispersion matches best with the visual extinction.

In addition to the velocity gradient, the line profiles clearly show an asymmetry which changes across the Globule. To quantify the asymmetry of the emission profiles we have calculated the 'skewness' of the emission at each position within the map. We follow Gregersen et al. (1997) and define skewness as a dimensionless ratio of the third moment to the $3 / 2$ power of the second moment where both are normalized by the first moment, such that

$$
\text { skewness }=\frac{\int T_{m b}(v)\left(v-v_{L S R}\right)^{3} d v}{\int T_{m b}(v) d v} /\left[\frac{\int T_{m b}(v)\left(v-v_{L S R}\right)^{2} d v}{\int T_{m b}(v) d v}\right]^{3 / 2}
$$

where $\mathrm{v}_{L S R}$ is the mean velocity of the Globule $\left(\sim-5.84 \mathrm{~km} \mathrm{~s}^{-1}\right)$. Figure 5 also shows the skewness image and reveals that, toward the west of the extinction ring, the profiles show a negative (blue) asymmetry, while toward the east, the profiles typically show a positive (red) asymmetry.

\subsection{Temperature and density tracers}

In addition to the $\mathrm{C}^{18} \mathrm{O}$ map, we have also obtained ${ }^{12} \mathrm{CO}$ and $\mathrm{CS}$ maps toward the Globule. While these results will be discussed in detail in Saul et al. (in prep), we include 
them here to help with the interpretation of the $\mathrm{C}^{18} \mathrm{O}$ results. Because ${ }^{12} \mathrm{CO}$ is typically optically thick, it can be used to trace the kinetic temperature of the gas. Because it has a large dipole moment, CS has a high critical density, and is typically excited collisionally only in high-density gas $\left(n \gtrsim 10^{5} \mathrm{~cm}^{-3}\right)$. Thus, the combination of ${ }^{12} \mathrm{CO}$ and $\mathrm{CS}$ can trace the warm, dense gas within the Globule.

Figure 6 shows the kinetic temperature map overlaid with contours of the smoothed visual extinction map. We find that the kinetic temperature displays a range of values over the Globule (from $\sim 8$ to $10 \mathrm{~K}$ ). The highest temperatures are not associated with the extinction ring, but are located to the north-west $\left(\mathrm{T}_{K}\right.$ of $9.6 \pm 0.5 \mathrm{~K}$ compared to $\sim 7.6 \pm$ $0.4 \mathrm{~K}$ in the south-east). The kinetic temperature of the gas associated with the extinction ring is $\sim 8.8 \pm 0.4 \mathrm{~K}$.

We find that the dense gas (as traced by the CS integrated intensity) is associated with the extinction ring (Fig. 17). While the morphology of the CS emission and extinction do not match exactly, there is a general correspondence between the two. However, the morphology of the CS emission agrees very well with the $\mathrm{C}^{18} \mathrm{O}$ emission: both peak on the western arc of the extinction ring. The fact that the $\mathrm{C}^{18} \mathrm{O}$ and $\mathrm{CS}$ emission match so well suggests that, chemically, this core does not appear to be evolved. Because carbon-chain molecules are considered 'early-time' molecules, they are abundant in chemically young cores and depleted in more evolved, star-forming cores. This is in contrast to molecules such as $\mathrm{N}_{2} \mathrm{H}^{+}$and $\mathrm{NH}_{3}$, which are considered 'late-time' molecules and, thus, are more abundant in chemically evolved cores (Bergin \& Langer 1997; Aikawa et al. 2005; Suzuki et al. 1992).

\section{Discussion}

\subsection{Comparison of the dust extinction to molecular abundances}

We find that the morphology of the $\mathrm{C}^{18} \mathrm{O}(1-0)$ integrated intensity generally matches the visual extinction. By smoothing the $\mathrm{A}_{V}$ image to match the angular resolution of the $\mathrm{C}^{18} \mathrm{O}$ map, we can make a point-by-point comparison between the integrated $\mathrm{C}^{18} \mathrm{O}$ emission and the visual extinction across the Globule.

Figure 8 shows the $\mathrm{C}^{18} \mathrm{O}$ integrated intensity as a function of $\mathrm{A}_{V}$. The individual data points are shown as small dots while the filled circles trace the overall trend and were generated by taking the median of the integrated intensities within evenly-spaced $A_{V}$ bins. Each $A_{V}$ bin is 0.7 mag wide; the errors bars in $A_{V}$ represent the range in each bin. The error bars in the integrated intensity represent the dispersion in the data. We find that the $\mathrm{C}^{18} \mathrm{O}$ integrated intensity is generally correlated with visual extinction across the complete 
range of measured extinction values. However, for any given $A_{V}$, there appears to be a range in the measured integrated intensities. Similar to what is observed toward other dark clouds, we find that $\mathrm{C}^{18} \mathrm{O}$ appears to be depleted only at the highest measured extinction $\left(\mathrm{A}_{V} \gtrsim 9.5 \mathrm{mag}\right)$. Figure 8 also shows a similar plot for the CS integrated intensity as a function of $\mathrm{A}_{V}$ and reveals that CS is also only depleted at the very highest extinctions. A similar analysis of the $\mathrm{C}^{18} \mathrm{O}$ integrated intensity as a function of $\mathrm{A}_{V}$ toward the denser core B68 (Bergin et al. 2002) shows that $\mathrm{C}^{18} \mathrm{O}$ is depleted for $\mathrm{A}_{V}>10$ mags. Thus, we do not see significant molecular depletion toward the Globule primarily because its mean column density is too low. The lack of a high density, centrally condensed core further suggests that the Globule is relatively young.

A least-squares fit to the $\mathrm{C}^{18} \mathrm{O}$ data (solid line) for $4 \mathrm{mag}<\mathrm{A}_{V}<9.5$ mag reveals that they are well matched to a linear fit with a constant slope over the complete range of measured extinction values. The derived linear fit is

$$
I\left(\mathrm{C}^{18} \mathrm{O}\right)=(0.17 \pm 0.01) A_{v}-0.04 \pm 0.07 \mathrm{~K} \mathrm{~km} \mathrm{~s}^{-1}
$$

The relative abundance of $\mathrm{C}^{18} \mathrm{O}$ to molecular hydrogen $\left(\mathrm{H}_{2}\right)$ can be estimated from comparisons between the derived values of $\mathrm{C}^{18} \mathrm{O}$ column density, $\mathrm{N}\left(\mathrm{C}^{18} \mathrm{O}\right)$, and $\mathrm{A}_{V}$. $\mathrm{A}$ least-squares linear fit to these data also show a linear correlation which is given by

$$
N\left(\mathrm{C}^{18} \mathrm{O}\right)=(1.4 \pm 0.1) \times 10^{14} A_{v}+(0.8 \pm 0.4) \times 10^{14} \mathrm{~cm}^{-2}
$$

Our derived slope is slightly lower than that calculated by Lada et al. (1994) within IC $5146\left(\sim 2.3 \times 10^{14} \mathrm{~cm}^{-2}\right)$ but is consistent with the range observed toward other dark clouds $\left(0.7-3.5 \times 10^{14} \mathrm{~cm}^{-2} \mathrm{mag}^{-1}\right.$; see Hayakawa et al. 1999; Harjunpää et al. 2004 and references therein). The tight correlation in the integrated intensity and, hence, $\mathrm{C}^{18} \mathrm{O}$ column density over all measured $\mathrm{A}_{V}$, suggests that there is little variation in the molecular abundance of $\mathrm{C}^{18} \mathrm{O}$ as a function of $\mathrm{A}_{V}$ and that the assumption of a single excitation temperature across the Globule is most likely valid.

By converting the measured $\mathrm{A}_{V}$ to a $\mathrm{H}_{2}$ column density, where $\mathrm{N}\left(\mathrm{H}_{2}\right) / \mathrm{A}_{V}=10^{21} \mathrm{~cm}^{-2} \mathrm{mag}^{-1}$, we can also derive the relation between the $\mathrm{C}^{18} \mathrm{O}$ and $\mathrm{H}_{2}$ column densities. A least-squares fit to plots of the data measured as $\mathrm{N}\left(\mathrm{C}^{18} \mathrm{O}\right)$ and $\mathrm{N}\left(\mathrm{H}_{2}\right)$ gives the relation

$$
N\left(\mathrm{C}^{18} \mathrm{O}\right)=(1.4 \pm 0.6) \times 10^{-7} N\left(H_{2}\right)-(0.8 \pm 0.4) \times 10^{14} \mathrm{~cm}^{-2}
$$

The derived slope is comparable to the value of $\sim 1.8 \times 10^{-7}$ calculated by Frerking et al. (1982) toward the dense cores within Taurus and $\rho$ Ophiuchus and to the values determined for the dense cores L 977 and FeSt 1-457 (Alves et al. 1999; Aguti et al. 2007). 
Even though we see very little overall $\mathrm{C}^{18} \mathrm{O}$ depletion toward the Globule, the apparent differences between the peaks of the visual extinction and $\mathrm{C}^{18} \mathrm{O}$ in the ring may arise as a result of depletion. Because the western arc of the ring has a higher mean extinction compared to the eastern arc, it is possible that the $\mathrm{C}^{18} \mathrm{O}$ is more depleted toward the western arc. These difference are seen in Figure 9 which plots the mean extinction, $\mathrm{C}^{18} \mathrm{O}$ integrated intensity, and the ratio of $\mathrm{C}^{18} \mathrm{O}$ integrated intensity to $\mathrm{A}_{V}$ across the Globule. For this analysis, the $\mathrm{A}_{V}$ image was smoothed to match the angular resolution of the $\mathrm{C}^{18} \mathrm{O}$. These plots were generated by taking the mean values in a $45^{\prime \prime}$ strip of constant Declination across the Globule. The enhanced extinction toward the ring is clearly seen, along with its central hole which is evident as an $\sim 10 \%$ decrement in the column density (top panel). This is in contrast to the $\mathrm{C}^{18} \mathrm{O}$ integrated intensity (middle panel) which does not show a significant decrement. These differences are most obvious in the ratio of the $\mathrm{C}^{18} \mathrm{O}$ integrated intensity to $\mathrm{A}_{V}$ (lower panel). We find that the ratio is essentially constant toward the western arc of the ring (positive offsets), but drops by $\sim 20 \%$ toward the eastern arc (negative offsets). This is consistent with the fact that depletion is typically only seen toward regions with the highest $\mathrm{A}_{V}$ (> 10 mags; e.g., Bergin et al. 2002). Because the mean $\mathrm{A}_{V}$ within most of the extinction ring is very close to this threshold, it may be that we are seeing the early onset of detectable $\mathrm{C}^{18} \mathrm{O}$ depletion in the western arc of the extinction ring. It is possible that more depletion is seen here simply because it has a higher column density.

This is consistent with a recent study of the solid-state $\mathrm{H}_{2} \mathrm{O}$ ice column densities toward the Globules within the Coalsack (Rodgers et al. 2007). Toward the outer edges of Globule 2 they find only a small amount of oxygen depletion. Because the time required to accrete the observed amount of oxygen is significantly shorter than the time for full oxygen depletion, they conclude that the Globule is relatively young $\left(\lesssim 10^{5}\right.$ years; Rodgers et al. 2007). The fact that this depletion time scale is comparable to the Globule's dynamical time scale (Lada et al. 2004) and the small amount of detectable depletion confirms the youth of the Globule.

\subsection{Global properties of the Globule}

While Globule 2 is one of the densest cores within the Coalsack Nebula, with a mean density $\mathrm{n}\left(\mathrm{H}_{2}\right)$ of $\sim 2.7 \times 10^{3} \mathrm{~cm}^{-3}$, it is less dense than typical cores found in Taurus $\left(\sim 1.2 \times 10^{4} \mathrm{~cm}^{-3}\right.$; Ladd \& Covev 2000) and the Pipe Nebula $\left(\sim 7.3 \times 10^{3} \mathrm{~cm}^{-3}\right.$; Lada et al. 2008). Because it is quiescent and does not harbor a central protostar, it is considered a dense, starless core. Moreover, the high Jeans mass to total mass ratio and the Bonnor-Ebert fit to the density profile for the outer regions of the Globule, suggest that the Globule is stable against col- 
lapse (Lada et al. 2004). However, the discovery of an extinction ring within the Globule has lead to speculations that it may be a dynamically unstable, transient core on the verge of condensing to form a protostar. With our $\mathrm{C}^{18} \mathrm{O}$ map, we can now test this idea and determine whether the Globule is gravitationally bound, in virial equilibrium, magnetically super-critical, rotating, thermally dominated, and/or pressure confined. These properties can reveal important clues to the nature and evolutionary status of the Globule.

\subsubsection{Gravitationally bound?}

From the single line profile toward Globule 2, Lada et al. (2004) suggested that the Globule is gravitationally bound. We now extend this analysis to include all the data measured toward the Globule. By comparing the escape velocity, $V_{e s c}$, to the average measured three-dimensional velocity dispersion across the Globule, we can determine if the Globule is gravitationally bound. The escape velocity is given by

$$
V_{e s c}=\sqrt{2 G M / R}
$$

where $\mathrm{M}$ and $\mathrm{R}$ are the Globule's mass and radius respectively. We use the values estimated from the dust extinction for the mass $\left(\mathrm{M}=6.1 M_{\odot}\right)$ and the radius calculated from the Bonnor-Ebert fit $\left(\mathrm{R}=290^{\prime \prime}, 0.21 \mathrm{pc}\right.$, or $6.51 \times 10^{13} \mathrm{~cm}$; Lada et al. 2004). Given these values, we estimate that the $\mathrm{V}_{\text {esc }}$ for Globule 2 is $0.50 \mathrm{~km} \mathrm{~s}^{-1}$.

The average three-dimensional velocity dispersion, $\sigma_{3 d}$, is calculated via the expression

$$
\sigma_{3 d}=\sqrt{3 a^{2}+3 \sigma_{n t}^{2}}
$$

where $a$ is the one-dimensional isothermal sound speed $\left(0.19 \mathrm{~km} \mathrm{~s}^{-1}\right.$ for hydrogen in a $10 \mathrm{~K}$ gas) and $\sigma_{n t}$ is the one-dimensional non-thermal velocity dispersion, i.e. $\sigma_{n t}=\sqrt{\sigma^{2}-\sigma_{t h}^{2}}$. The measured velocity dispersion, $\sigma$, was estimated from the average in the second moment map across the Globule and is $\sim 0.18 \mathrm{~km} \mathrm{~s}^{-1}$. Assuming the gas is at $10 \mathrm{~K}$, the one-dimensional thermal velocity dispersion, $\sigma_{t h}$, is $0.05 \mathrm{~km} \mathrm{~s}^{-1}$. Thus, the non-thermal velocity dispersion, $\sigma_{n t}$, is $\sim 0.17 \mathrm{~km} \mathrm{~s}^{-1}$ and implies that the turbulent motions are subsonic. Using these values we find that the average three-dimensional velocity dispersion, $\sigma_{3 d}$, is $0.44 \mathrm{~km} \mathrm{~s}^{-1}$. Because $\sigma_{3 d}$ is formally less than the escape velocity, it is likely that the Globule is indeed gravitationally bound, although only marginally so.

The one-dimensional virial velocity dispersion, $\sigma_{\text {virial }}$, was also calculated via the expression 


$$
\sigma_{\text {virial }}=\sqrt{\frac{1}{5} \frac{G M}{R}}
$$

and was found to be $0.16 \mathrm{~km} \mathrm{~s}^{-1}$. This is lower than both the sound speed in a $10 \mathrm{~K}$ gas $\left(0.19 \mathrm{~km} \mathrm{~s}^{-1}\right)$ and the measured $\mathrm{C}^{18} \mathrm{O}$ velocity dispersion across the Globule $\left(0.18 \mathrm{~km} \mathrm{~s}^{-1}\right)$. The fact that the $\sigma_{\text {virial }}$ is lower than the measured velocity dispersions indicates that the Globule is not virialized. Thus, it appears that the Globule is only marginally bound and not in virial equilibrium, implying that is it extremely young.

\subsubsection{Magnetically super-critical?}

The role of magnetic support within the Globule can be measured via the ratio of the observed mass to magnetic flux $(\Phi)$ represented in terms of the critical value. We follow Crutcher (2007) and define this ratio as

$$
\lambda=\frac{(M / \Phi)_{\text {observed }}}{(M / \Phi)_{\text {critical }}}=7.6 \times 10^{-21} \frac{N\left(H_{2}\right)}{B}
$$

where $\mathrm{N}\left(\mathrm{H}_{2}\right)$ is in $\mathrm{cm}^{-2}$ and $\mathrm{B}$ is in $\mu \mathrm{G}$. To calculate the magnetic field, $\mathrm{B}$, we use the Chandrasekhar \& Fermi (1953) equation

$$
B=\frac{\sigma_{v}}{\sigma_{\Theta}} \sqrt{4 \pi \rho}=10 \sqrt{\frac{n\left(H_{2}\right)}{2.7 \times 10^{3}}} \mu G
$$

where $\sigma_{v}$ is the one-dimensional velocity dispersion and $\sigma_{\Theta}$ is the dispersion in the measured polarization angles. Using $\sigma_{v}=0.18 \mathrm{~km} \mathrm{~s}^{-1}, \sigma_{\Theta}=0.66 \mathrm{rad}$ (Jones et al. 1984), and a molecular hydrogen density, $\mathrm{n}\left(\mathrm{H}_{2}\right)$, of $2.7 \times 10^{3} \mathrm{~cm}^{-3}$, we find that $\mathrm{B} \sim 10 \mu \mathrm{G}$. Thus, the directly observed

ratio, $\lambda_{\text {observed }}$, is $\sim 4$.8. Applying corrections for geometrical effects of $1 / 3$ (Crutcher 2007), we find that $\lambda$ is $\sim 1$.4. A value of $\lambda$ greater than unity implies that the magnetic field is super-critical. Thus, we find that the Globule is marginally super-critical which suggests that the Globule is not dominated by magnetic support and that it is likely bound if not already in the process of gravitational contraction.

\subsubsection{Rotating?}

As mentioned previously, the channel maps, $(\ell-V)$ diagram, and first moment map (the intensity weighted velocity field) all show signs of a velocity gradient across the Globule. In 
the $\mathrm{C}^{18} \mathrm{O}$ first moment map (Fig. 5), the lines of constant velocity are nearly parallel and are aligned north-south. If the velocity gradient is linear when projected on the sky, we can relate the observed velocity of any point on the projected surface of the Globule to the velocity gradient via the expression (Goodman et al. 1993; Lada et al. 2003)

$$
v_{L S R}=v_{0}+\frac{d v}{d s} \Delta \alpha \cos \theta+\frac{d v}{d s} \Delta \delta \sin \theta
$$

where $\Delta \alpha$ and $\Delta \delta$ are the offsets in Right Ascension and Declination (in arcsec), $d v / d s$ is the magnitude of the velocity gradient in the plane of the sky, $\theta$ is its direction (measured from east of north), and $v_{0}$ is the systemic radial velocity of the Globule. The magnitude and direction of the velocity gradient and the Globule's systemic velocity were determined through a least-squares fit to the observed $\mathrm{C}^{18} \mathrm{O}$ velocity distribution (as shown in Fig. 5). We find that $d v / d s=1.00 \pm 0.01 \mathrm{~km} \mathrm{~s}^{-1} \mathrm{pc}^{-1}, \theta=90.1 \pm 0.3^{\circ}$, and $v_{0}=-5.81 \pm 0.01 \mathrm{~km} \mathrm{~s}^{-1}$. This velocity gradient is very similar to that found in the $\mathrm{C}^{18} \mathrm{O}$ survey of the Coalsack Nebula by Kato et al. (1999). Even though these data were under-sampled (2'.7 beam on a $4^{\prime}$ grid), they reveal a velocity gradient across Globule 2 of $\sim 0.90 \mathrm{~km} \mathrm{~s}^{-1} \mathrm{pc}^{-1}$. If the Globule was undergoing solid-body rotation whose axis is perpendicular to the line-of-sight, then this velocity gradient would correspond to an angular velocity of $2.9 \times 10^{-14} \mathrm{~s}^{-1}$ (Kato et al. 1999).

It is possible that the velocity gradient arises due to the rotation of the Globule. Assuming solid-body rotation, the ratio of rotational kinetic energy to gravitational potential energy, $\beta$, can be used to determine the significance of rotation to the overall dynamics of the Globule. Following Goodman et al. (1993), we calculate $\beta$ via the expression

$$
\beta=\frac{(1 / 2) I w^{2}}{q G M^{2} / R}=\frac{1}{2} \frac{p}{q} \frac{w^{2} R^{3}}{G M}
$$

where $M$ and $R$ are the mass and radius of the Globule respectively, the moment of inertia is given by $I=p M R^{2}, q$ is defined such that the gravitational potential energy is $q G M^{2} / R$, and $w=(d v / d s) / \sin i$. We assume a sphere with $\mathrm{a}^{-2}$ density profile, which implies $p / q$ is 0.22 , and that $\sin i=1$. Using $\mathrm{M}=6.1 M_{\odot}, \mathrm{R}=6.51 \times 10^{17} \mathrm{~cm}$, and $d v / d s=1.00 \mathrm{~km} \mathrm{~s}^{-1} \mathrm{pc}^{-1}$, we find that $\beta=0.04$. This value of $\beta$ is similar to other cores: for example, Goodman et al. (1993) find that most clouds have $\beta \leq 0.05$.

Thus, we calculate that the apparent rotation does not contribute significantly to the overall stability of Globule 2. In any event, it appears that the observed dynamics of the Globule are more complicated than simply solid body rotation and it is likely, therefore, that the observed velocity gradient is not tracing a coherent rotating structure. Nevertheless, the energy in the velocity gradient is unimportant relative to gravity. 


\subsubsection{Thermally dominated?}

The one-dimensional $\mathrm{C}^{18} \mathrm{O}$ velocity dispersions $\left(\sigma \sim 0.18 \mathrm{~km} \mathrm{~s}^{-1}\right)$ are $\sim 3.6$ times broader than what would be expected if the profiles were purely dominated by thermal motions. This suggests that significant non-thermal motions characterize the velocity field of the gas and that turbulence may play a role in the internal pressure of the Globule. To quantify the degree to which turbulence may be contributing to the internal pressure, we calculate the ratio of the thermal to non-thermal (turbulent) pressure as given by

$$
R_{p}=\frac{a^{2}}{\sigma_{n t}^{2}}
$$

where $a$ is the one-dimensional isothermal sound speed $\left(0.19 \mathrm{~km} \mathrm{~s}^{-1}\right.$ for hydrogen in a $10 \mathrm{~K}$ gas) and $\sigma_{n t}$ is the one-dimensional non-thermal velocity dispersion. Taking the average $\sigma_{n t}$ across the Globule, we estimate the ratio of thermal to non-thermal pressure, $\mathrm{R}_{p}$, is $\sim 1.2$. Thus, it appears that thermal pressure is comparable to, or slightly greater than, the non-thermal, or turbulent, pressure within the Globule.

While it is useful to calculate average values across the Globule, there are clear differences in the $\mathrm{C}^{18} \mathrm{O}$ emission within and either side of the extinction ring. Indeed, if we take the typical measured velocity dispersion across the extinction ring and compare it to two regions on opposing sides of the ring, we find that the velocity dispersion toward the ring ( $\sigma$ of $0.20 \pm 0.01 \mathrm{~km} \mathrm{~s}^{-1}$ ) is $\sim 1.3$ times greater than that observed outside the ring $\left(0.15 \pm 0.01\right.$ and $0.17 \pm 0.01 \mathrm{~km} \mathrm{~s}^{-1}$ to the east and west of the ring respectively). Using these values we find that the non-thermal velocity dispersions $\left(\sigma_{n t}\right)$ in and out of the ring are 0.19 and $\sim 0.15 \mathrm{~km} \mathrm{~s}^{-1}$ respectively. Thus, we see turbulent sonic motions within the ring and turbulent subsonic motions external to it.

The differences between these regions are most obvious when we consider the ratio of the thermal to turbulent pressures, $R_{p}$ : within the ring, $R_{p}$ is $\sim 1$, while external to the ring $R_{p}$ is $\sim 1.6$. This suggests that thermal pressure is more dominant external to the ring, whereas toward the ring, the contributions from thermal and turbulent pressure are comparable.

This can also be seen in the left panels of Figure 10 which shows the $\mathrm{C}^{18} \mathrm{O}$ non-thermal velocity dispersion $\left(\sigma_{n t}\right)$ and the ratio of thermal to non-thermal pressure $\left(\mathrm{R}_{p}\right)$ as a function of $\mathrm{A}_{V}$ for all positions in the map. In the upper panel the dotted horizontal line marks the one-dimensional isothermal sound speed. In the lower panel the dotted horizontal line marks the point at which thermal and turbulent pressure support are equal. The dashed vertical line marks the approximate visual extinction at the edge of the extinction ring ( $\sim 9.5$ mags). These plots show that the general trend is for higher non-thermal velocity dispersions, $\sigma_{n t}$, 
and lower values for $\mathrm{R}_{p}$ as $\mathrm{A}_{V}$ increases. Coincident with the extinction ring $\sigma_{n t}$ transitions from subsonic to trans-sonic and $\mathrm{R}_{p}$ transitions from greater than to less than 1 . Thus, it appears that the outer regions of the Globule are thermally dominated, while toward the extinction ring non-thermal pressure may be more important.

\subsubsection{Pressure confined?}

To further characterize its nature, we have also calculated the internal gas pressures within the Globule. The total pressure within the Globule is measured by a combination of the pressures due to thermal $\left(\mathrm{P}_{t h}\right)$ and non-thermal motions $\left(\mathrm{P}_{n t}\right)$. The thermal and non-thermal pressures were calculated via the expressions

$$
P_{t h}=\rho a^{2} \quad \text { and } \quad P_{n t}=\rho \sigma_{n t}^{2}
$$

where $\rho$ is the mean density, $\rho=3 M /\left(4 \pi R^{3}\right)$, $a$ is the one-dimensional isothermal sound speed, and $\sigma_{n t}$ is the one-dimensional non-thermal velocity dispersion. We find that $\mathrm{P}_{t h} / k \sim$ $2.8 \times 10^{4} \mathrm{~K} \mathrm{~cm}^{-3}$ and $\mathrm{P}_{n t} / k \sim 2.2 \times 10^{4} \mathrm{~K} \mathrm{~cm}^{-3}$. Thus, the total pressure, $\mathrm{P}_{\text {total }} / k$, due to thermal and non-thermal motions is $\sim 5.0 \times 10^{4} \mathrm{~K} \mathrm{~cm}^{-3}$.

Because the Globule lies within the larger Coalsack molecular cloud complex, it is possible that the weight of the Coalsack molecular cloud may also be a significant source of confining pressure. The pressure due to the weight of the cloud, $\mathrm{P}_{\text {cloud }}$, is given by

$$
P_{\text {cloud }}=\frac{3 \pi}{20} G \Sigma^{2} \phi_{G}
$$

where $G$ is the Gravitational constant, $\Sigma$ is the mean mass surface density of the cloud $\left(\Sigma=M / \pi R^{2}\right)$, and $\phi_{G}$ is a dimensionless correction factor to account for the non-spherical geometry of a cloud (Bertoldi \& McKee 1992). Given the Coalsack molecular cloud has a mass of $\sim 3550 M_{\odot}$, radius of $\sim 7.1$ pc (Nyman et al. 1989), and assuming $\phi_{G}=1.6$, we find that $\mathrm{P}_{\text {cloud }}=0.8 \times 10^{4} \mathrm{~K} \mathrm{~cm}^{-3}$.

This pressure is more than a factor of 5 lower than the internal pressure calculated from a combination of the thermal and non-thermal motions which suggests that it is unlikely that the weight of the Coalsack molecular cloud contributes significantly to the overall confining pressure of the Globule. Interestingly, this pressure is comparable to the pressure resulting from the interstellar medium, $\mathrm{P}_{I S M} / k \sim 10^{4} \mathrm{~K} \mathrm{~cm}^{-3}$ (Bertoldi \& McKee 1992). Thus, we find that the total internal pressure is greater than both $\mathrm{P}_{I S M} / k$ and $\mathrm{P}_{\text {cloud }} / k$ and that the Globule is super-critical. This adds to earlier suggestions that the Globule is gravitationally bound. This is in contrast to dense cores found within the Pipe Nebula most of which are 
thought to be thermally dominated, unbound cores that are confined by external pressure (Lada et al. 2008) and suggests that the Globule is bound by gravity.

\subsection{The Origin of the Ring}

\subsubsection{External pressure driving a compression wave?}

Recent dynamical models for Globule 2 (Hennebelle et al. 2006) suggest that the ring structure has formed due to a rapid increase in the external pressure on a core with very little initial turbulence. In this picture, the ring structure traces the dense regions swept up by the compression wave as it propagates through the core. The central depression exists because the compression wave has not yet converged in the center. The models generated by Hennebelle et al. (2006) predict that toward the center of the Globule the line profiles should be double-peaked, while toward the outer edges of the Globule the profiles should show a single line.

With our $\mathrm{C}^{18} \mathrm{O}$ map we can now test this model. Due to the receding and approaching compression wave, the Hennebelle et al. (2006) model predicts that positions within 50" of the center should show a double-peaked line profile. Figure 11 shows a comparison between our data and the model of Hennebelle et al. (2006) for different impact parameters, $b$, across the Globule. The spectra were generated by averaging over annuli at the impact parameters $b=0,0.04,0.08$ and $0.12 \mathrm{pc}$. The example model spectra were estimated from figure 2 of Hennebelle et al. (2006) and are plotted so that the center velocity (their $\mathrm{V}=0.0 \mathrm{~km} \mathrm{~s}^{-1}$ ) corresponds to the $\mathrm{v}_{L S R}$ of the Globule $\left(-5.8 \mathrm{~km} \mathrm{~s}^{-1}\right)$. The individual line centers, widths and relative intensities were taken from their figure, while the peak temperatures were scaled to approximately match the data.

From this comparison we find that at larger impact parameters $(b=0.08$ and $0.12 \mathrm{pc})$ the models match the data well. These regions correspond to the edges of the Globule, off the extinction ring. Toward the ring, however, we find the models differ considerably from the data. While we do observe a double-line profile at impact parameters $b<0.04 \mathrm{pc}$, the separation of the line centers and the relative intensities of the two components are noticeably different from what the models predict. Toward the center of the ring $(b=0.0 \mathrm{pc})$, we find that the two components are separated by $\sim 0.24 \mathrm{~km} \mathrm{~s}^{-1}$, with the red component dominating. Here the models predict the line profiles should be separated by $1 \mathrm{~km} \mathrm{~s}^{-1}$ and have the same relative intensity. Similarly, at an impact parameter $b=0.04 \mathrm{pc}$ (which completely encompasses the extinction ring), we also see a dominant red component separated by $\sim 0.21 \mathrm{~km} \mathrm{~s}^{-1}$ from the fainter blue component. 
The discrepancies between the observed line profiles and the model predictions could arise simply because of the assumptions of a symmetric compression wave. The apparent fragmentation within, and the non-symmetrical nature of, the extinction ring suggests that the mechanism that has given rise to the ring is unlikely to be smooth and symmetric. Moreover, the predicted line separations could be higher than that measured due to an overestimation of the speed at which the material is imploding within the models. While the model predicts the integrated intensity and velocity dispersion should increase within the extinction ring, it fails to predict the velocity gradient that we observe. It is possible that the Globule may be rotating slightly while undergoing an implosion due to a non-symmetric increase in external pressure. More detailed modeling with the inclusion of a non-symmetric compression wave is necessary to test this idea.

\subsubsection{The merging of two subsonic flows?}

Although we cannot definitively rule out that the ring may have formed via an external compression wave, the above model in its current form is unable to completely characterize the observed kinematics within this Globule. Because the extinction ring correlates with the $\mathrm{C}^{18} \mathrm{O}$ velocity dispersion, and, thus, appears organized, it is unlikely that the extinction ring is simply a chance super-position of material along the line of sight.

Rather than the implosion of the Globule due to an increase in its external pressure, it appears that we may be seeing the shearing, or merging, of two separate velocity features. Gaussian fits to the spectra across the Globule suggests there are two components that arise within the ring; one at $\sim-5.7 \mathrm{~km} \mathrm{~s}^{-1}$ and the other at $\sim-5.9 \mathrm{~km} \mathrm{~s}^{-1}$ (see Fig. 3). The emission in these channels, shown in Figure 2, reveals that while both of these components peak within the ring, they also show emission external to, and on opposing sides of, the extinction ring. Because both of these components have non-thermal subsonic one-dimensional velocity dispersions, we speculate that these components correspond to two distinct subsonic flows of turbulent gas that are merging in the center of the Globule.

Of all the measured parameters, we find the spatial match between the one-dimensional velocity dispersion and the visual extinction to be the best. Thus, the convergence of these two flows within the extinction ring has potentially caused the enhanced column density. It may be, therefore, that the extinction ring is neither a ring nor shell seen in projection, but simply arises due to the merging of molecular gas and dust as the two flows are interacting. Thus, the increase in the velocity dispersion and non-thermal pressure toward the extinction ring may simply reflect the fact that two flows are interacting here. Because the outer regions of the Globule appear thermally dominated and stable against collapse, it may be that the 
Globule is evolving from the outside in and has yet to stabilize, as evidenced by the lack of a centrally condensed core.

If the two flows were indeed interacting within the ring, then we would also expect to see an increase in the $\mathrm{C}^{18} \mathrm{O}$ integrated intensity toward the ring: in the absence of temperature, opacity and depletion effects, higher velocity dispersions should translate directly into higher integrated intensities. Although a general increase in the integrated intensity is clearly seen toward the ring, it is interesting to note that the $\mathrm{C}^{18} \mathrm{O}$ emission and extinction do not peak at the same location within the ring. Figures 5 and 7 show that the $\mathrm{C}^{18} \mathrm{O}$ and $\mathrm{CS}$ integrated emission peaks toward western arc of the ring whereas the extinction and velocity dispersion peak toward the eastern arc. These morphological differences can be explained in terms of molecular depletion at high extinctions. Figure 8 shows that both the $\mathrm{C}^{18} \mathrm{O}$ and $\mathrm{CS}$ are depleted for $\mathrm{A}_{V}>9.5$ mags. Because the eastern arc is dominated by extinctions $>9.5$ mags it shows a higher degree of molecular depletion compared to the western arc.

As mentioned previously, there is a temperature gradient across the Globule that goes from a $\mathrm{T}_{K}$ of $9.6 \pm 0.5 \mathrm{~K}$ in the north-west to $\sim 7.6 \pm 0.4 \mathrm{~K}$ in the south-east. Thus, it appears that the two distinct velocity flows may have slightly different temperatures. Moreover, the fact that the temperature does not increase significantly within the ring, supports the idea that it has not formed due to the propagation of a shock front through the Globule. If this were the case, we would expect to see the temperature increase in the shock front and be coincident with the ring.

\section{Conclusions}

Globule 2 is one of the densest cores within the Coalsack Nebula, but contains no evidence for star-formation. Despite the fact that overall it appears stable against collapse, a recent high angular resolution extinction map reveals Globule 2 contains a central ring of enhanced column density and, thus, may in fact be a dynamically unstable, transient core on the verge of condensing to form a protostar. It has been speculated that this central ring of column density has formed either as a result of turbulence or from a shock induced via an external compression wave (Lada et al. 2004; Hennebelle et al. 2006).

Here we have combined dust extinction measurements with a high angular resolution $\mathrm{C}^{18} \mathrm{O}$ molecular line map in order to study the detailed kinematics of Globule 2. The $\mathrm{C}^{18} \mathrm{O}$ map reveals that the Globule is marginally gravitationally bound and not virialized. Moreover, the $\mathrm{C}^{18} \mathrm{O}$ map reveals a complex velocity profile that appears to arise from two distinct velocity features that are present both toward the ring and external to it. In addition, 
these velocity features appear to trace material on opposing sides of the extinction ring.

Each of these velocity flows have non-thermal subsonic velocity dispersions. Outside the extinction ring the Globule is thermally dominated, however, both the measured velocity dispersion and non-thermal pressure increase toward the extinction ring. Of all the measured properties, we find the best spatial correspondence between the one-dimensional velocity dispersion and the visual extinction. We suggest, therefore, that the extinction ring is an enhancement in column density due to the merging of the two subsonic flows. Thus, it appears as though we are seeing the convergence of two subsonic flows within a bound object. Moreover, because the outer regions of the Globule appear stable against collapse and there is no centrally condensed core, it may be that the Globule is evolving from the outside in and has yet to stabilize.

Finally, although we cannot entirely rule out recent dynamical models for the formation

of the extinction ring via an external compression wave (Hennebelle et al. 2006), these models are unable to completely characterize the observed $\mathrm{C}^{18} \mathrm{O}$ kinematics. The discrepancies between the model and the data may arise because of the simplified assumption of a spherical compression wave.

We thank the referee, Dr. Whitworth, for a thorough reading of the paper and the useful suggestions which have improved the paper considerably. We also thank Alyssa Goodman and Scott Schnee for providing us with the IDL gradient fitting code.

\section{REFERENCES}

Aguti, E. D., Lada, C. J., Bergin, E. A., Alves, J. F., \& Birkinshaw, M. 2007, ApJ, 665, 457

Aikawa, Y., Herbst, E., Roberts, H., \& Caselli, P. 2005, ApJ, 620, 330

Alves, J., Lada, C. J., \& Lada, E. A. 1999, ApJ, 515, 265

Alves, J., Lada, C. J., Lada, E. A., Kenyon, S. J., \& Phelps, R. 1998, ApJ, 506, 292

Bergin, E. A., Alves, J., Huard, T., \& Lada, C. J. 2002, ApJ, 570, L101

Bergin, E. A. \& Langer, W. D. 1997, ApJ, 486, 316

Bertoldi, F. \& McKee, C. 1992, ApJ, 395, 140

Bok, B. J. 1977, Nature, 266, 145 
Bourke, T. L., Hyland, A. R., Robinson, G., James, S. D., \& Wright, C. M. 1995, MNRAS, 276,1067

Broderick, A. E., Keto, E., Lada, C. J., \& Narayan, R. 2007, ApJ, 671, 1832

Brooks, J. W., Sinclair, M. W., \& Manefield, G. A. 1976, MNRAS, 175, 117

Cambrésy, L. 1999, A\&A, 345, 965

Chandrasekhar, S. \& Fermi, E. 1953, ApJ, 118, 113

Crutcher, R. M. 2007, EAS Publications Series, 23, 37

Frerking, M. A., Langer, W. D., \& Wilson, R. W. 1982, ApJ, 262, 590

Goodman, A. A., Benson, P. J., Fuller, G. A., \& Myers, P. C. 1993, ApJ, 406, 528

Gregersen, E. M., Evans, II, N. J., Zhou, S., \& Choi, M. 1997, ApJ, 484, 256

Harjunpää, P., Lehtinen, K., \& Haikala, L. K. 2004, A\&A, 421, 1087

Hayakawa, T., Mizuno, A., Onishi, T., Hara, A., Yamaguchi, R., \& Fukui, Y. 1999, PASJ, 51,919

Hennebelle, P., Whitworth, A. P., \& Goodwin, S. P. 2006, A\&A, 451, 141

Jones, T. J., Hyland, A. R., \& Bailey, J. 1984, ApJ, 282, 675

Jones, T. J., Hyland, A. R., Robinson, G., Smith, R., \& Thomas, J. 1980, ApJ, 242, 132

Kato, S., Mizuno, N., Asayama, S.-i., Mizuno, A., Ogawa, H., \& Fukui, Y. 1999, PASJ, 51, 883

Keto, E., Broderick, A. E., Lada, C. J., \& Narayan, R. 2006, ApJ, 652, 1366

Lada, C. J., Bergin, E. A., Alves, J. F., \& Huard, T. L. 2003, ApJ, 586, 286

Lada, C. J., Huard, T. L., Crews, L. J., \& Alves, J. F. 2004, ApJ, 610, 303

Lada, C. J., Lada, E. A., Clemens, D. A., \& Bally, J. 1994, ApJ, 429, 694

Lada, C. J., Muench, A. A., Rathborne, J., Alves, J. F., \& Lombardi, M. 2008, ApJ, 672, 410

Ladd, E. F. \& Covey, K. R. 2000, ApJ, 536, 380 
Ladd, N., Purcell, C., Wong, T., \& Robertson, S. 2005, Publications of the Astronomical Society of Australia, 22, 62

Maret, S., Bergin, E. A., \& Lada, C. J. 2007, ApJ, 670, L25

Matsumoto, T. \& Hanawa, T. 2003, ApJ, 595, 913

Nyman, L.-A., Bronfman, L., \& Thaddeus, P. 1989, A\&A, 216, 185

Racca, G., Gómez, M., \& Kenyon, S. J. 2002, AJ, 124, 2178

Redman, M. P., Keto, E., \& Rawlings, J. M. C. 2006, MNRAS, 370, L1

Rodgers, S. D., Charnley, S. B., Smith, R. G., \& Butner, H. M. 2007, MNRAS, 379, 807

Sault, R. J., Teuben, P. J., \& Wright, M. C. H. 1995, in Astronomical Society of the Pacific Conference Series, Vol. 77, Astronomical Data Analysis Software and Systems IV, ed. R. A. Shaw, H. E. Payne, \& J. J. E. Hayes, 433--

Suzuki, H., Yamamoto, S., Ohishi, M., Kaifu, N., Ishikawa, S.-I., Hirahara, Y., \& Takano, S. 1992, ApJ, 392, 551

Tapia, S. 1973, in IAU Symposium, Vol. 52, Interstellar Dust and Related Topics, ed. J. M. Greenberg \& H. C. van de Hulst, 43-- 


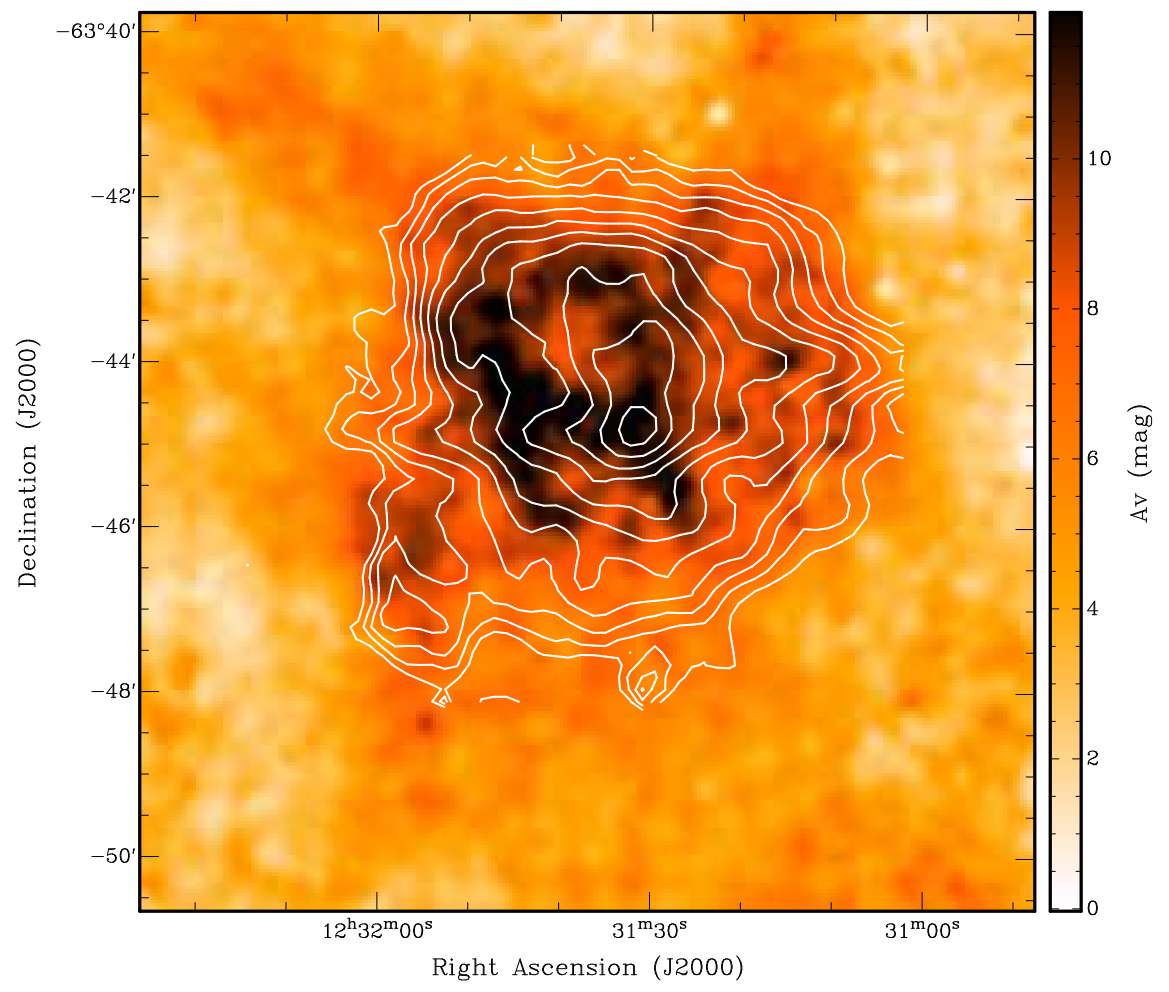

Fig. 1. - The visual extinction $\left(\mathrm{A}_{V}\right)$ map of Globule 2 in the Coalsack Nebula $\left(15^{\prime \prime}\right.$ angular resolution; Lada et al. 2004) in color scale overlaid with contours of the $\mathrm{C}^{18} \mathrm{O}$ integrated intensity (46" angular resolution). The emission was integrated over the velocity range of -7.0 to $-5.0 \mathrm{~km} \mathrm{~s}^{-1}$. The contour levels are from 1.0 to 2.0 in steps of $0.1 \mathrm{~K} \mathrm{~km} \mathrm{~s}^{-1}$. Note that the general morphology of the $\mathrm{C}^{18} \mathrm{O}$ integrated intensity follows the enhanced extinction associated with the ring. 
$-6.19 \mathrm{~km} \mathrm{~s}^{-1}$

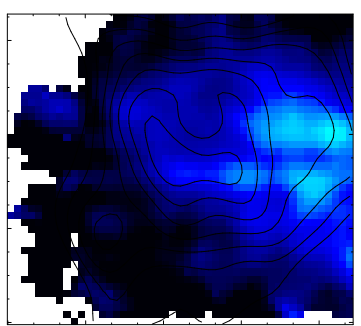

$-5.82 \mathrm{~km} \mathrm{~s}^{-1}$

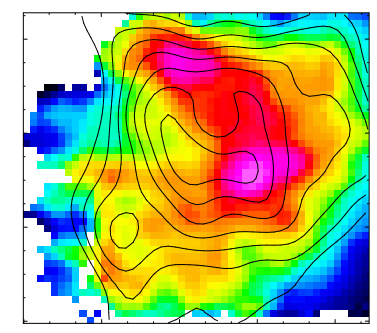

$-6.10 \mathrm{~km} \mathrm{~s}^{-1}$

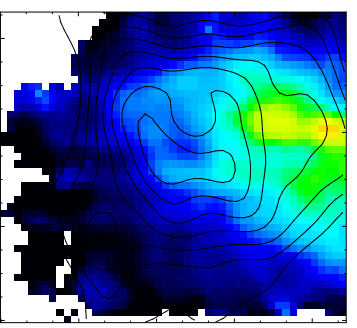

$-5.73 \mathrm{~km} \mathrm{~s}^{-1}$

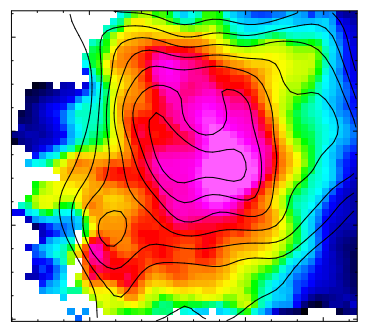

$-6.01 \mathrm{kms}^{-1}$

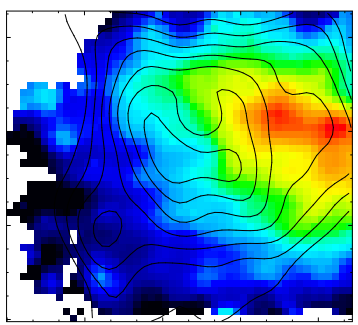

$-5.64 \mathrm{kms}^{-1}$

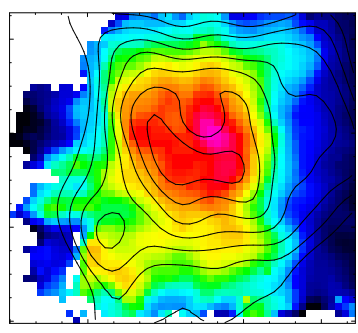

$-5.91 \mathrm{~km} \mathrm{~s}^{-1}$

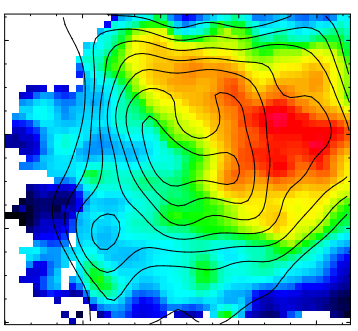

$-5.55 \mathrm{~km} \mathrm{~s}^{-1}$

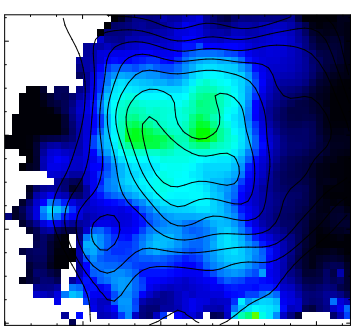

Fig. 2.- $\mathrm{C}^{18} \mathrm{O}(1-0)$ channel maps overlaid with contours of the smoothed visual extinction map toward Globule 2 (46" angular resolution). The contour levels are from 6 to 12 in steps of 0.75 magnitudes. The color maps show a single channel centered at the $\mathrm{v}_{L S R}$ marked above each image. All images cover the same extent in Right Ascension and Declination $(\sim$ $\left.6^{\prime} \times 6^{\prime}\right)$ and have the same color scale from a $\mathrm{T}_{m b}$ of 0 (black) to $3.4 \mathrm{~K}$ (pink). We see a clear velocity gradient across the Globule. 


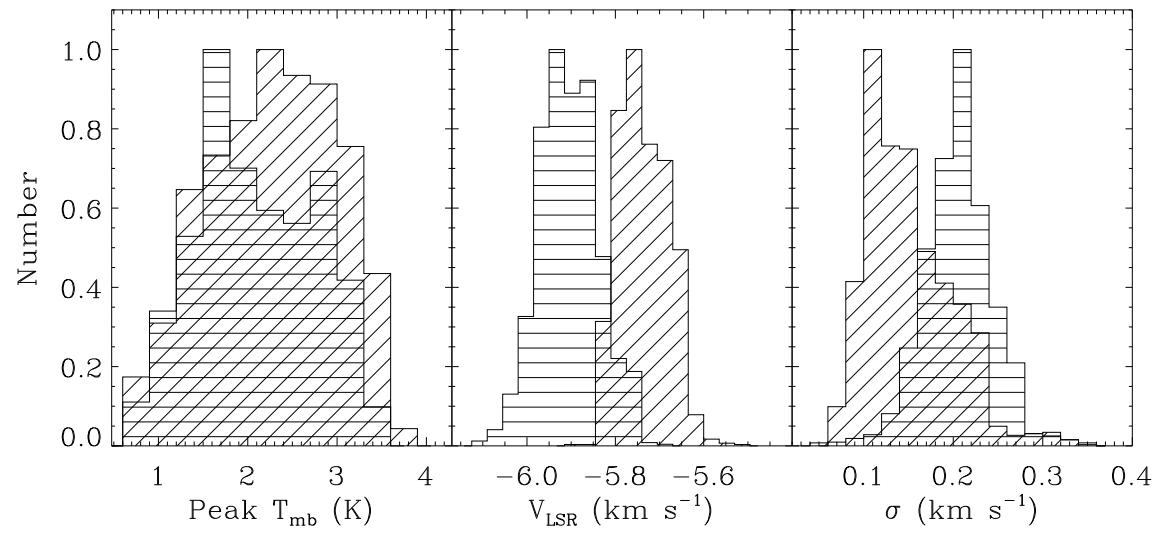

Fig. 3.- Normalized number distributions for the peak temperature $\left(\mathrm{T}_{m b}\right)$, central velocity $\left(\mathrm{v}_{L S R}\right)$, and one-dimensional velocity dispersion $(\sigma)$ output from the Gaussian fitting procedure. In all cases the histograms with horizontal lines represent the data for the 'broad' line component, while histograms with diagonal lines represent the 'narrow' line component. Note that the two components show a range in their central $\mathrm{v}_{L S R}$, suggesting the kinematics of the Globule are more complicated than two simple Gaussian emission profiles. 

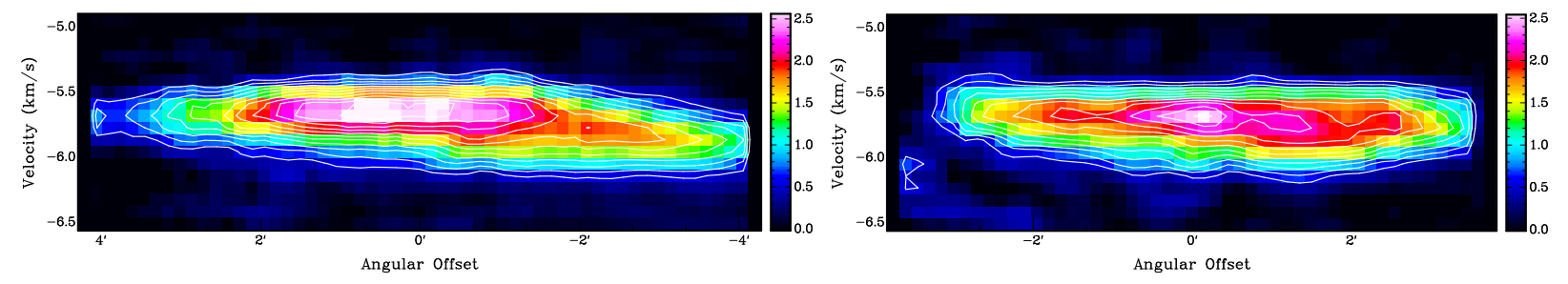

Fig. 4.- $\mathrm{C}^{18} \mathrm{O}(1-0)$ position-velocity, $(\ell-V)$, diagram (color-scale and contours). The emission was averaged over the extinction ring in both Declination (left) and Right Ascension (right). A velocity gradient is most apparent in the left panel (the Declination-averaged position-velocity diagram) which is not surprising given this direction is roughly perpendicular to the axis of the apparent gradient in the first moment map (see Fig. (5)). 

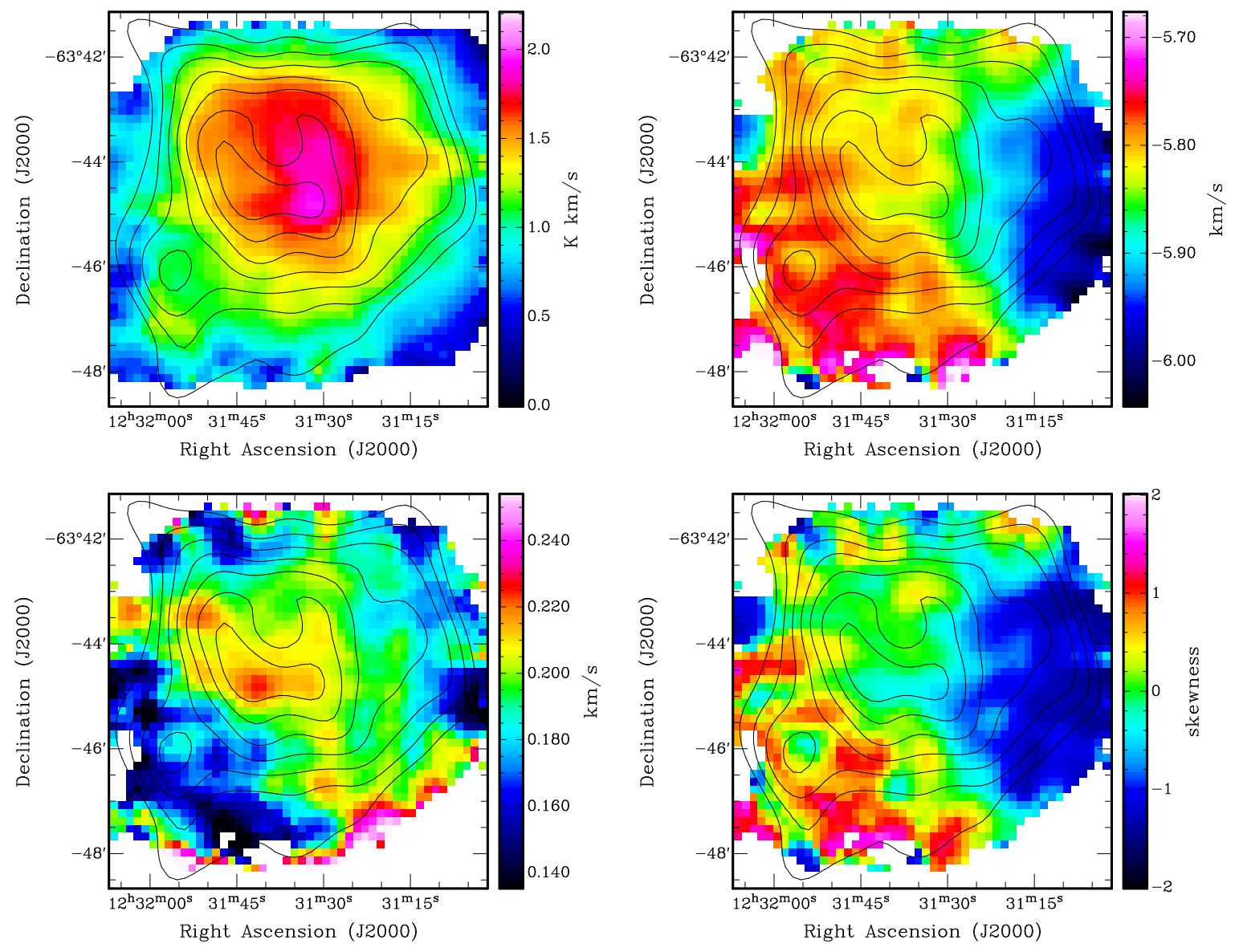

Fig. 5.- $\mathrm{C}^{18} \mathrm{O}(1-0)$ moment maps in color scale with contours of the smoothed extinction image. Top left: Zeroth moment (integrated intensity). Top right: First moment (intensity weighted velocity field). Bottom left: Second moment (velocity dispersion). Bottom right: Skewness image. The contour levels are from 6 to 12 in steps of 0.75 magnitudes. The extinction ring is seen as an enhancement in the $\mathrm{C}^{18} \mathrm{O}$ integrated intensity. The first moment map reveals a velocity gradient across the Globule. The velocity dispersion measured by the second moment map clearly increases toward the extinction ring. The skewness image reveals that the profiles change from a negative (blue) asymmetry to a positive (red) asymmetry across the Globule. 


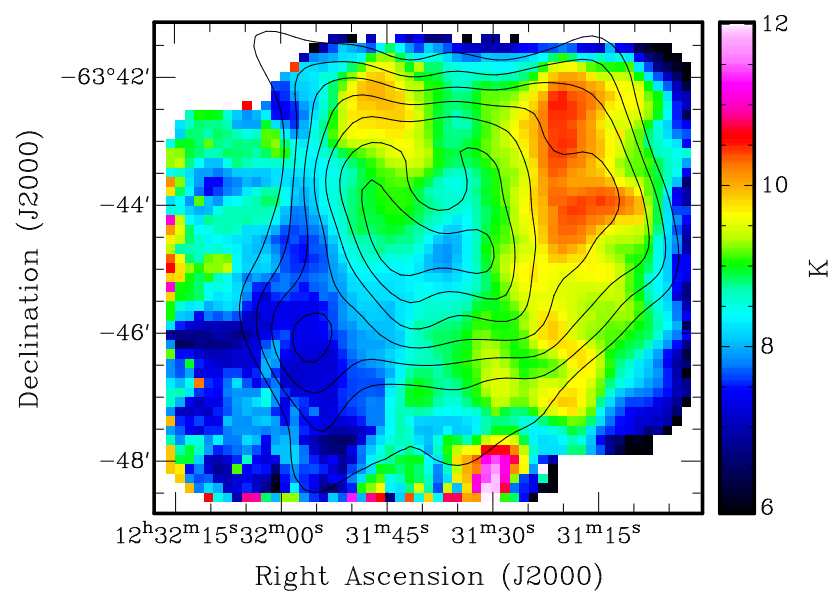

Fig. 6. - Kinetic temperature $\left(\mathrm{T}_{K}\right)$ across the Globule derived from the optically thick ${ }^{12} \mathrm{CO}$ emission. Overlaid are contours of the smoothed visual extinction image (levels are from 6 to 12 in steps of 0.75 magnitudes). We see a temperature gradient across the Globule of 9.6 $\pm 0.5 \mathrm{~K}$ in the north-west to $\sim 7.6 \pm 0.4 \mathrm{~K}$ in the south-east. 


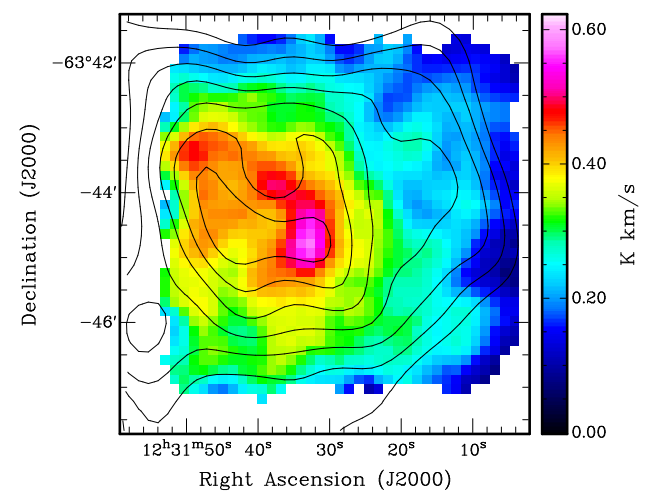

Fig. 7.- CS integrated intensity image overlaid with contours of the smoothed visual extinction image. The contour levels are from 6 to 12 in steps of 0.75 magnitudes. Bright CS emission is seen coincident with the extinction ring, however, their overall morphologies are different. The morphology of the CS integrated intensity emission matches very well the $\mathrm{C}^{18} \mathrm{O}$ integrated intensity. 

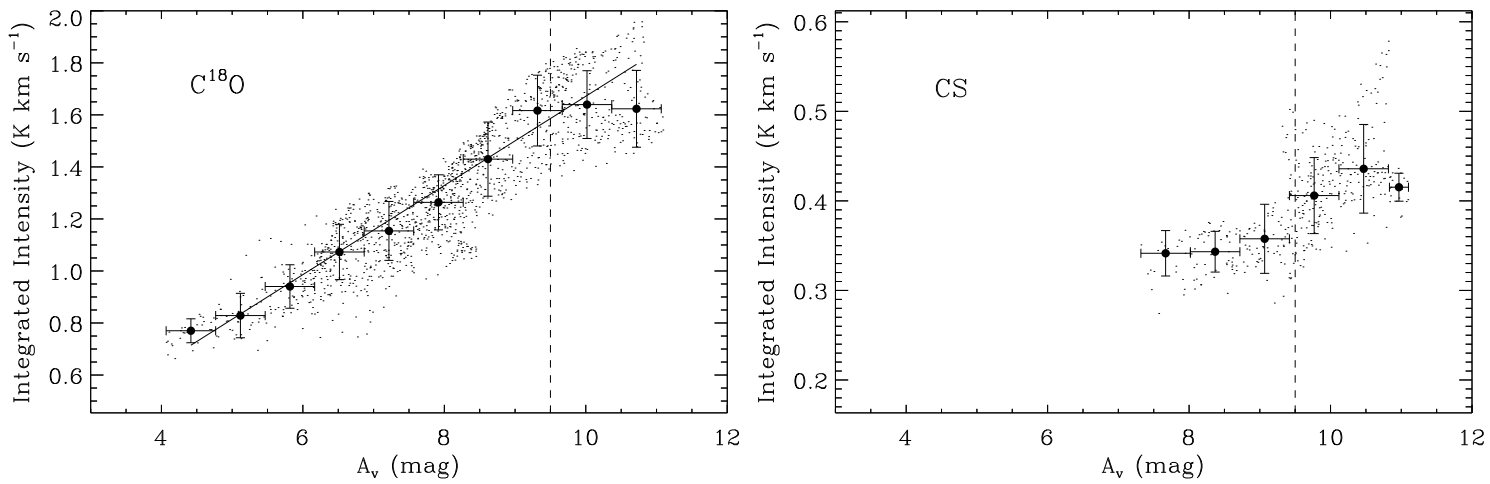

Fig. 8.- $\mathrm{C}^{18} \mathrm{O}$ (left) and $\mathrm{CS}$ (right) integrated intensity as a function of visual extinction $\left(\mathrm{A}_{V}\right)$ for all positions across the Globule. The individual data points are shown as small dots while the filled circles were generated by taking the median of the integrated intensities within evenly-spaced $A_{V}$ bins (0.7 mags). The errors bars in $A_{V}$ represent the range in each bin. The error bars in the integrated intensity represent the dispersion in the data. The dashed vertical line marks the approximate visual extinction at the edge of the extinction ring $\left(\sim 9.5\right.$ mags). The solid line is a least-squares fit to the data for 4 mags $<\mathrm{A}_{V}<9.5$ mags. We see a general correlation between the $\mathrm{C}^{18} \mathrm{O}$ and $\mathrm{CS}$ integrated intensity and $\mathrm{A}_{V}$ over the complete range in $\mathrm{A}_{V}$, however, we also note that both the $\mathrm{C}^{18} \mathrm{O}$ and $\mathrm{CS}$ appear depleted at high extinctions $\left(\mathrm{A}_{V}>9.5\right.$ mags $)$. 


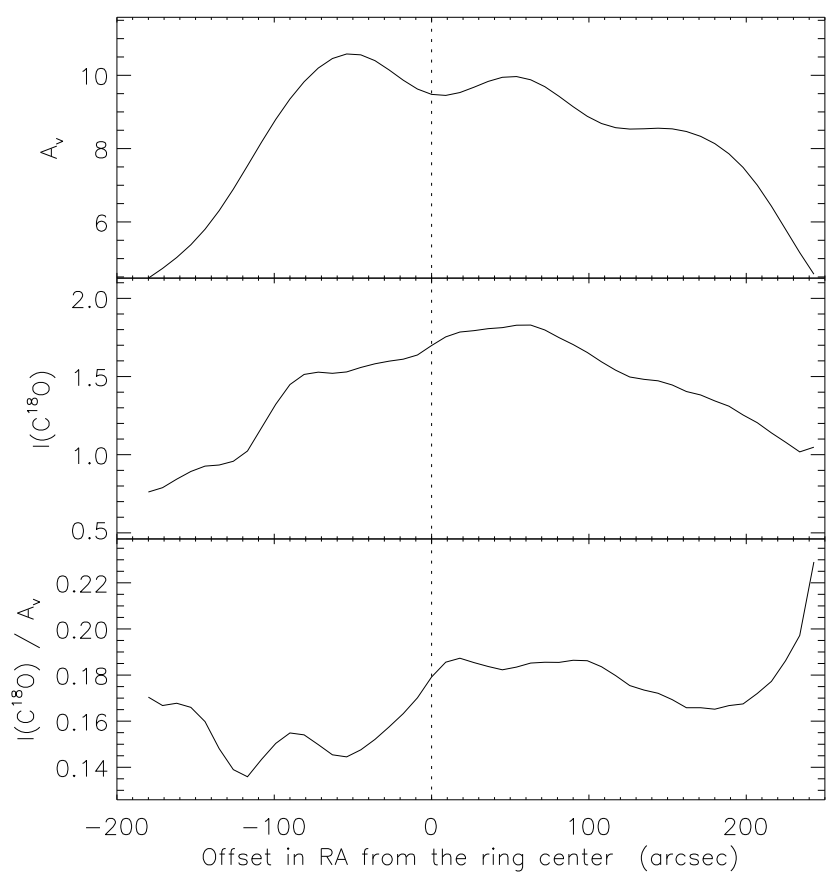

Fig. 9.- Visual extinction $\left(\mathrm{A}_{V}\right), \mathrm{C}^{18} \mathrm{O}$ integrated intensity, and the ratio of $\mathrm{C}^{18} \mathrm{O}$ integrated intensity to $\mathrm{A}_{V}$ across the Globule. These plots were generated by taking the mean values in a $45^{\prime \prime}$ strip of constant Declination across the Globule. The $\mathrm{A}_{V}$ across the Globule shows the enhanced extinction toward the ring and its central $10 \%$ decrement. The $\mathrm{C}^{18} \mathrm{O}$ integrated intensity does not show a similar decrement. The lower panel shows that the ratio is essentially constant toward the western arc of the ring (positive offsets), but drops by $20 \%$ toward the eastern arc (negative offsets). 

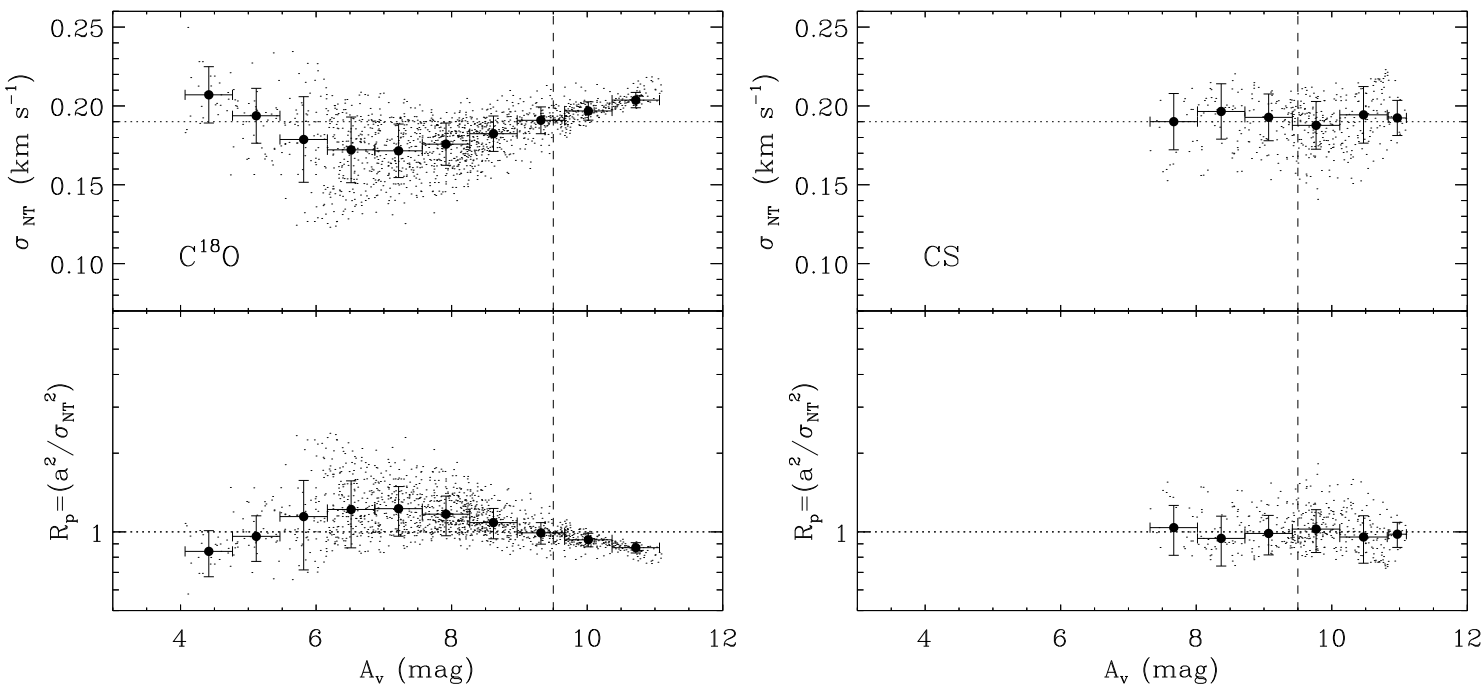

Fig. 10.- Measured non-thermal velocity dispersion ( $\sigma_{n t}$; upper panels) and the ratio of thermal to non-thermal pressures ( $\mathrm{R}_{p}$; lower panels) as a function of $\mathrm{A}_{V}$ for all positions with the Globule ( $\mathrm{C}^{18} \mathrm{O}$ left, $\mathrm{CS}$ right). The individual data points are shown as small dots while the filled circles were generated by taking the median of the integrated intensities within evenly-spaced $A_{V}$ bins. Each $A_{V}$ bin is 0.7 mag wide; the errors bars in $A_{V}$ represent the range in each bin. The error bars in $\sigma_{n t}$ and $\mathrm{R}_{p}$ represent the dispersion in the data. In the upper panels the dotted horizontal line marks the one-dimensional isothermal sound speed (0.19 $\mathrm{km} \mathrm{s}^{-1}$ for hydrogen in a $10 \mathrm{~K}$ gas). In the lower panels the dotted horizontal line marks the point at which thermal and turbulent pressure are equal. The dashed vertical line marks the approximate visual extinction at the edge of the extinction ring $(\sim 9.5$ mags $)$. For $\mathrm{C}^{18} \mathrm{O}$, we see that as $\mathrm{A}_{V}$ increases the non-thermal velocity dispersion increases while the ratio of thermal to non-thermal pressure decreases. Coincident with the ring $\sigma_{n t}$ transitions from subsonic to trans-sonic and $\mathrm{R}_{p}$ transitions from greater than to less than 1 . This suggests that thermal pressure dominates external to the extinction ring and that turbulence may be more important within the ring. In contrast, $\sigma_{n t}$ and $\mathrm{R}_{p}$ appear constant across the Globule as traced by the CS emission. 

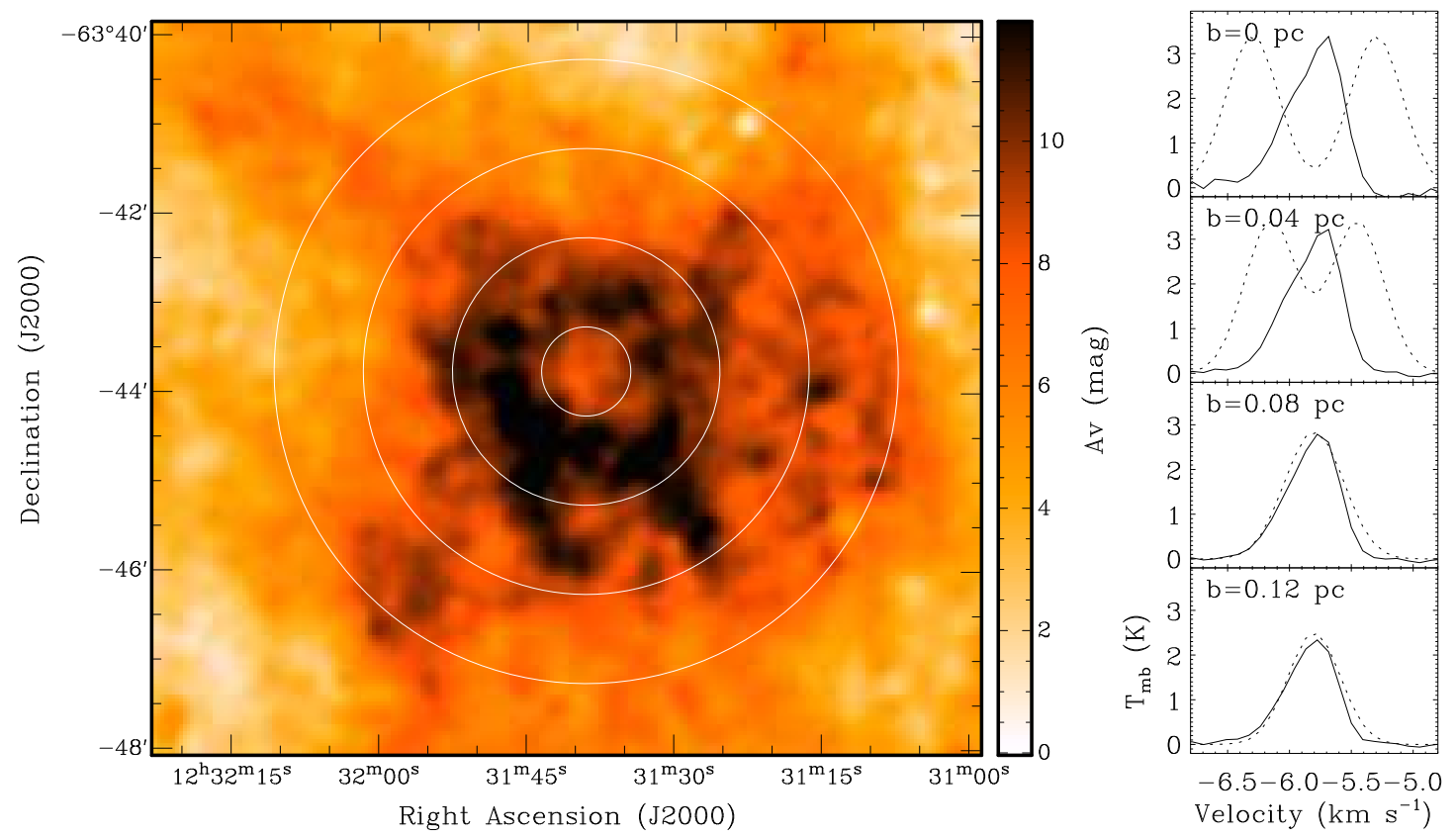

Fig. 11. - Comparison between our $\mathrm{C}^{18} \mathrm{O}$ data and the models of Hennebelle et al. (2006) for different impact parameters, $b$, across the Globule. The spectra (right panels) were generated by averaging over annuli at the impact parameters $b=0,0.04,0.08$ and $0.12 \mathrm{pc}$ (shown as circles on the extinction image: left panel). The example model spectra were estimated from figure 2 of Hennebelle et al. (2006) and are plotted so that the center velocity (their $\mathrm{V}=0.0 \mathrm{~km} \mathrm{~s}^{-1}$ ) corresponds to the $\mathrm{v}_{L S R}$ of the Globule $\left(-5.8 \mathrm{~km} \mathrm{~s}^{-1}\right)$. The individual line centers, widths, and relative intensities were taken from their figure, while the peak temperatures were scaled to approximately match the data. We find that at larger impact parameters $(b>0.08 \mathrm{pc})$ the model matches the data well. Toward the ring $(b<0.04 \mathrm{pc})$, however, we see noticeable differences between the data and the model which might arise from the initial assumptions of the simple model (i.e. inflow speeds and a symmetric compression wave). 